\title{
Integration of dynamic contrast-enhanced magnetic resonance imaging and T2-weighted imaging radiomic features by a canonical correlation analysis-based feature fusion method to predict histological grade in ductal breast carcinoma
}

To cite this article before publication: Ming Fan et al 2019 Phys. Med. Biol. in press https://doi.org/10.1088/1361-6560/ab3fd3

\section{Manuscript version: Accepted Manuscript}

Accepted Manuscript is "the version of the article accepted for publication including all changes made as a result of the peer review process, and which may also include the addition to the article by IOP Publishing of a header, an article ID, a cover sheet and/or an 'Accepted Manuscript' watermark, but excluding any other editing, typesetting or other changes made by IOP Publishing and/or its licensors"

This Accepted Manuscript is ( 2019 Institute of Physics and Engineering in Medicine.

During the embargo period (the 12 month period from the publication of the Version of Record of this article), the Accepted Manuscript is fully protected by copyright and cannot be reused or reposted elsewhere.

As the Version of Record of this article is going to be / has been published on a subscription basis, this Accepted Manuscript is available for reuse under a CC BY-NC-ND 3.0 licence after the 12 month embargo period.

After the embargo period, everyone is permitted to use copy and redistribute this article for non-commercial purposes only, provided that they adhere to all the terms of the licence https://creativecommons.org/licences/by-nc-nd/3.0

Although reasonable endeavours have been taken to obtain all necessary permissions from third parties to include their copyrighted content within this article, their full citation and copyright line may not be present in this Accepted Manuscript version. Before using any content from this article, please refer to the Version of Record on IOPscience once published for full citation and copyright details, as permissions will likely be required. All third party content is fully copyright protected, unless specifically stated otherwise in the figure caption in the Version of Record.

View the article online for updates and enhancements. 


\title{
Integration of Dynamic Contrast-enhanced Magnetic Resonance
}

Imaging and T2-weighted Imaging Radiomic Features by a

Canonical Correlation Analysis-based Feature Fusion Method to

Predict Histological Grade in Ductal Breast Carcinoma

Ming Fan ${ }^{1}$, Zuhui $\mathrm{Liu}^{1}$, Sudan $\mathrm{Xie}^{1}$, Maosheng $\mathrm{Xu}^{2}$, Shiwei Wang ${ }^{2}$, Xin $\mathrm{Gao}^{3}$ and Lihua $\mathrm{Li}^{1 *}$

${ }^{1}$ Institute of Biomedical Engineering and Instrumentation, Hangzhou Dianzi University, Hangzhou, China

${ }^{2}$ Department of Radiology, First Affiliated Hospital of Zhejiang Chinese Medical University, Hangzhou, Zhejiang, China

${ }^{3}$ King Abdullah University of Science and Technology (KAUST), Computational Bioscience Research Center (CBRC), Computer, Electrical and Mathematical Sciences and Engineering Division (CEMSE), Thuwal, 23955-6900, Saudi Arabia.

*Corresponding Author Email: lilh@ @du.edu.cn

\begin{abstract}
Tumour histological grade has prognostic implications in breast cancer. Tumour features in dynamic contrast-enhanced magnetic resonance imaging (DCE-MRI) and T2-weighted (T2W) imaging can provide related and complementary information in the analysis of breast lesions to improve MRI-based histological status prediction in
\end{abstract}


breast cancer. A dataset of 167 patients with invasive ductal carcinoma (IDC) was assembled, consisting of 72 low/intermediate-grade and 95 high-grade cases with preoperative DCE-MRI and T2W images. The data cohort was separated into development $(n=111)$ and validation $(n=56)$ cohorts. Each tumour was segmented in the precontrast and the intermediate and last postcontrast DCE-MR images and was mapped to the tumour in the $\mathrm{T} 2 \mathrm{~W}$ images. Radiomic features, including texture, morphology, and histogram distribution features in the tumour image, were extracted for those image series. Features from the DCE-MR and T2W images were fused by a canonical correlation analysis (CCA)-based method. The support vector machine (SVM) classifiers were trained and tested on the development and validation cohorts, respectively. SVM-based recursive feature elimination (SVM-RFE) was adopted to identify the optimal features for prediction. The areas under the ROC curves (AUCs) for the T2W images and the DCE-MRI series of precontrast, intermediate and last postcontrast images were $0.750 \pm 0.047,0.749 \pm 0.047$, and $0.788 \pm 0.045$, respectively, for the development cohort and $0.715 \pm 0.068,0.704 \pm 0.073$, and $0.744 \pm 0.067$, respectively, for the validation cohort. After the CCA-based fusion of features from the DCE-MRI series and T2W images, the AUCs increased to $0.751 \pm 0.065$, $0.803 \pm 0.0600$ and $794 \pm 0.060$ in the validation cohort. Moreover, the method of fusing features between DCE-MRI and T2W images using CCA achieved better performance than the concatenation-based feature fusion or classifier fusion methods. Our results demonstrated that anatomical and functional MR images yield complementary information, and feature fusion of radiomic features by matrix transformation to 
optimize their correlations produced a classifier with improved performance for predicting the histological grade of IDC.

Keywords - Ductal breast carcinoma, Dynamic contrast-enhanced magnetic resonance imaging (DCE-MRI), T2-weighted imaging, Canonical correlation analysis (CCA)

\section{INTRODUCTION}

Invasive carcinomas are morphologically subdivided according to growth pattern and degree of differentiation by assessing histological type and histological grade, respectively (Rakha et al., 2010). Studies have demonstrated that tumour grade is an independent prognostic factor in specific subgroups of breast cancer patients, including oestrogen receptor (ER)-positive breast cancer patients who have not received neoadjuvant chemotherapy (Ehinger et al., 2017; Wirapati et al., 2008) and those who have (Ellis et al., 2008). The histological grade may have different prognostic implications for different histological types (Engstrom et al., 2015). Invasive ductal carcinoma (IDC), also known as infiltrating ductal carcinoma, is the most common histological type of breast cancer, accounting for approximately $80 \%$ of all breast cancers. Therefore, accurate identification of tumour histological grade in IDC can provide useful guidance for prognosis in this histological type.

Dynamic contrast-enhanced magnetic resonance imaging (DCE-MRI) is an important tool because of its suitability for measuring tumour vascularity and 
assessing the effectiveness of novel antiangiogenic and antivascular agents ( $\mathrm{Li}$ et al., 2015). Studies have performed DCE-MRI-based radiomic analysis for the prediction of molecular subtypes (Fan et al., 2017a; Li et al., 2016), gene expression (Wu et al., 2017) and neoadjuvant chemotherapy response in breast cancer patients (Braman et al., 2017; Fan et al., 2017b). Related studies have shown that DCE-MRI parameters are strongly associated with histological grade in breast cancer (Burnside et al., 2016; Montemurro et al., 2007). The results from a DCE-MRI study suggest that spiculated tumour margins are correlated with lower histological grade, while rim enhancement is correlated with high histological grade in breast cancer (Chang et al., 2009). A recent study by Nam et al. found that high peak enhancement was significantly associated with a high $\mathrm{T}$ stage, high clinical stage, and high histological grade (Nam et al., 2018). Although the studies relating DCE-MRI features to histological characteristics showed many encouraging results, their specificities varied.

For the above reasons, researchers have investigated different methods, including the use of T2-weighted (T2W) images along with DCE-MRI images, for identifying associations with tumour characteristics (e.g., diagnosis of breast lesions) (Kuhl et al., 1999). T2W images can be used to detect oedema, haemorrhage, mucus, and cystic fluid within a lesion, which is valuable for the T2W MRI evaluation of breast lesions and complementary to DCE-MRI examination (Kuhl et al., 1999). Many research efforts have focused on combinations of T2W MRI and T1-weighted MRI or diffusion-weighted imaging in the analysis of breast lesions (Bhooshan et al., 2011; Westra et al., 2014) to improve breast MRI computer-aided diagnosis (CAD) 
(Gallego-Ortiz and Martel, 2017) and the prediction of sentinel lymph node (SLN) metastasis in breast cancer (Dong et al., 2018). Combining information from these multiparametric images yields better performance than that of any single image, as verified in various studies (Parekh and Jacobs, 2017; Zhang et al., 2017). However, whether and how the predictions of the histological grade from DCE-MRI could be improved by integrating T2W and DCE-MRI features remains unclear. Moreover, these combinatory predictions were independently implemented via feature concatenation or by averaging output prediction scores from several different parametric image classifiers and are therefore omitted to assess the correlated information from these images.

We assume that tumour features in anatomical and functional MR images from the same source could represent related and complementary information. As an effective feature fusion technique, canonical correlation analysis (CCA) has been widely applied in many fields, including facial recognition (Wang et al., 2015b), data regression analysis (Abraham and Merola, 2005), and neurodegenerative disease identification (Smith et al., 2015; Zhao et al., 2017). This analysis captures covariations between two datasets that are then mixed into one compound variable.

Therefore, in this study, we investigated the accuracy of multiparametric image fusion with $\mathrm{T} 2 \mathrm{~W}-$ and DCE-MRI-based radiomic features by CCA for the prediction of histological grade in IDC.

\section{MATERIALS AND METHODS}

\subsection{Overview of the Framework}


In this study, radiomic analysis of DCE-MRI series and T2W images was used to develop a model for predicting histological grade in IDC (Figure 1). Tumours were segmented on DCE-MRI scans and aligned to T2W image scans, and the features, including morphological features, texture features, and histogram distribution of tumour images, were extracted. We assumed that multiparametric images from a tumour area could have certain correlations, and CCA identifies a matrix transformation for optimizing the correlations between features from DCE-MRI and T2W images. Therefore, the DCE-MRI series of the precontrast, intermediate postcontrast and last postcontrast scans was integrated with $\mathrm{T} 2 \mathrm{~W}$ images, and the following feature fusion methods were included and compared with respect to histological grade prediction in IDC: 1) feature-level fusion by CCA, which takes advantage of the correlations between different parametric images; 2) feature-level fusion via concatenation, which simply concatenates features from different images; and 3) decision-level fusion by averaging the classifier scores using an equal weighting factor.

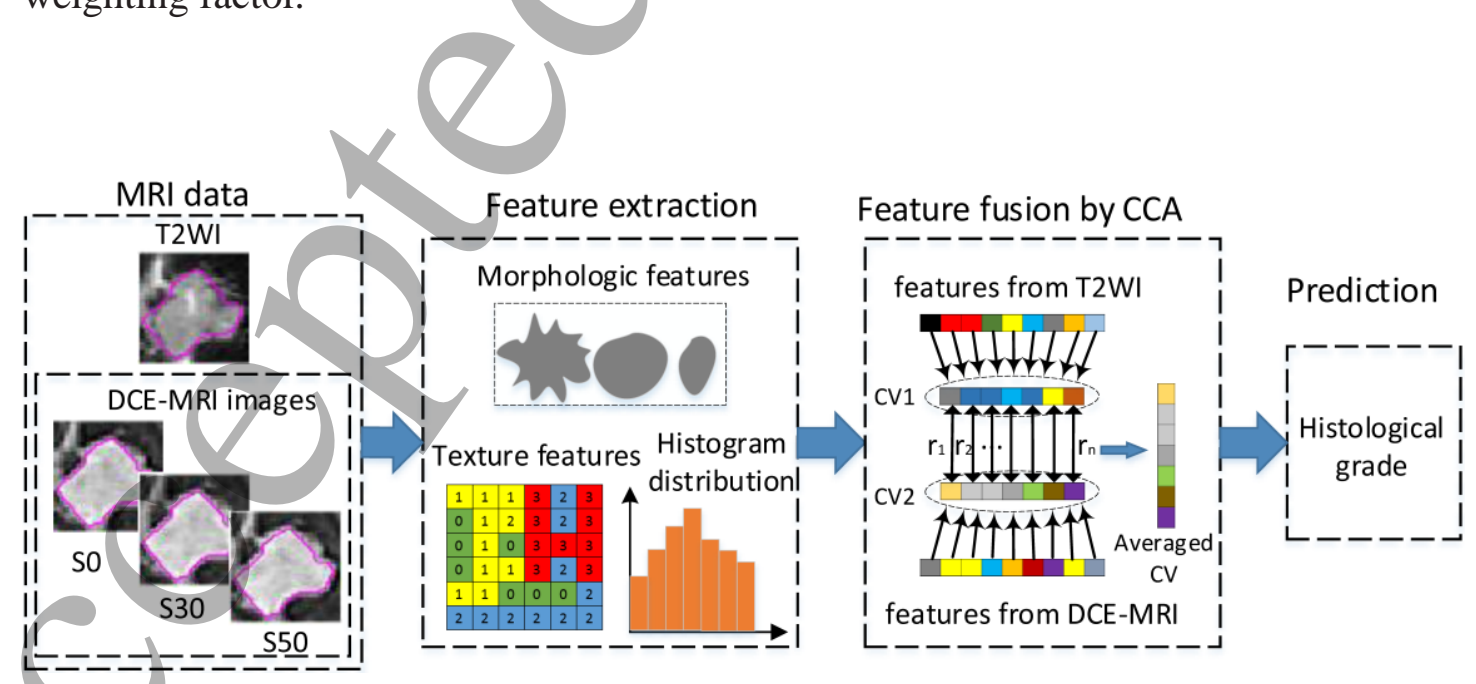

Figure 1. Overview of the framework. CV, canonical variable 


\subsection{Data Cohort}

This study was approved by the Internal Research Review and Ethical Committee of the First Affiliated Hospital of Zhejiang Chinese Medical University. The need for informed consent was waived due to the retrospective nature of this study. The initial dataset included 207 samples with MRIs and the corresponding clinical information. We excluded 23 patients who did not have an IDC and 14 patients who did not have T2W image scans. We also removed the data of 3 patients for whom a complete DCE-MRI series was not available. The final dataset included 167 samples from IDC patients, specifically 5 low-grade, 67 intermediate-grade and 95 high-grade breast cancer patients, resulting in 72 low/intermediate-grade (43.11\%) and 95 high-grade (56.89\%) patients. The clinical information for these cases is shown in Table 1. The samples were randomly separated into two independent cohorts, the development $(111 / 167,66.5 \%)$ and the validation cohorts $(56 / 167,33.5 \%)$. Among these, 48 out of 111 samples in the development cohort had low/intermediate-grade disease, while 24 out of 57 patients had high-grade disease. The statistical analysis revealed no significant differences $(p>0.05)$ regarding age; oestrogen receptor $(E R)$, progesterone receptor (PR), and human epidermal growth factor receptor 2 (HER2) positivity; Ki-67; menopausal status; and maximum diameter between the development and validation cohorts. 
Table 1. Characteristics of patients in the development and validation cohorts

\begin{tabular}{|c|c|c|c|c|c|c|}
\hline Characteristic & All $(n=167)$ & $\begin{array}{l}\text { Low/intermedi } \\
\text { ate grade } \\
(n=72)\end{array}$ & $\begin{array}{l}\text { High grade } \\
(\mathrm{n}=95)\end{array}$ & $\begin{array}{l}\text { Development } \\
\text { cohort }(n=111)\end{array}$ & $\begin{array}{l}\text { Validation } \\
\text { cohort } \\
(n=56)\end{array}$ & \\
\hline $\mathrm{PR}^{\mathrm{b}}$ & & & & & & 0.979 \\
\hline Positive & 103 & $55 / 72(32.9 \%)$ & $49 / 95(29.3 \%)$ & $63 / 111(37.7 \%)$ & $40 / 56(24.0 \%)$ & \\
\hline Negative & 64 & $17 / 72(10.2 \%)$ & $46 / 95(27.5 \%)$ & $48 / 111(28.7 \%)$ & $16 / 56(9.6 \%)$ & \\
\hline $\mathrm{ER}^{\mathrm{b}}$ & & & & & & 0.914 \\
\hline Positive & 109 & $57 / 72(34.1 \%)$ & $52 / 95(31.1 \%)$ & $69 / 111(41.3 \%)$ & $40 / 56(24.0 \%)$ & \\
\hline Negative & 58 & $15 / 72(9.0 \%)$ & $43 / 95(25.7 \%)$ & $42 / 111(25.1 \%)$ & $16 / 56(9.6 \%)$ & \\
\hline $\mathrm{Ki}-67^{\mathrm{b}}$ & & & & & & 0.304 \\
\hline High & 119 & $44 / 72(26.3 \%)$ & $75 / 95(44.9 \%)$ & $81 / 111(48.5 \%)$ & $38 / 56(22.7 \%)$ & \\
\hline Low & 48 & $28 / 72(16.8 \%)$ & $20 / 95(12.0 \%)$ & $30 / 111(18.0 \%)$ & $18 / 56(10.8 \%)$ & \\
\hline HER $2 ~^{\text {b }}$ & & & & & & 0.576 \\
\hline Positive & 33 & $11 / 72(6.6 \%)$ & $22 / 95(13.2 \%)$ & $22 / 111(13.2 \%)$ & $11 / 56(6.6 \%)$ & \\
\hline Negative & 134 & $6172(36.5 \%)$ & $73 / 95(43.7 \%)$ & $89 / 111(53.3 \%)$ & $45 / 56(26.9 \%)$ & \\
\hline $\begin{array}{l}\text { Menopausal } \\
\text { status }^{\text {b }}\end{array}$ & & & & & & 0.706 \\
\hline Premenopausal & 92 & $42 / 72(25.1 \%)$ & $50 / 95(30.0 \%)$ & $60 / 111(35.9 \%)$ & $32 / 56(19.2 \%)$ & \\
\hline Postmenopausal & 75 & $30 / 72(18.0 \%)$ & $45 / 95(26.9 \%)$ & $51 / 111(30.5 \%)$ & $24 / 56(14.4 \%)$ & \\
\hline $\begin{array}{l}\text { Maximum } \\
\text { diameter }^{\mathrm{c}}(\mathrm{mm})\end{array}$ & $23.75(6-100)$ & $18.18(6-40)$ & $27.97(10-100)$ & $23.61(6-100)$ & $24.03(7-50)$ & 0.835 \\
\hline $\operatorname{Age}^{\mathbf{c}}(\mathrm{y})$ & $\begin{array}{l}51.90 \\
(28-83)\end{array}$ & $51.53(28-83)$ & $52.19(31-82)$ & $52.05(28-83)$ & $51.63(33-70)$ & 0.800 \\
\hline
\end{tabular}

ap-value for the low- versus high-grade tumour comparison.

bData were evaluated using the chi-square test.

cData were assessed using analysis of variance (ANOVA).

$\mathrm{ER}=$ oestrogen receptor, $\mathrm{PR}=$ progesterone receptor. HER2=human epidermal growth factor receptor 2; Numbers in parentheses are percentages.

\subsection{MR Protocol}

All breast MR examinations were performed with the patient placed in the prone position, using a 3.0-T imager (Siemens Medical Systems, Erlangen, Germany) equipped with a dedicated eight-channel breast array coil. The parameters for T2WI were as follows: repetition time (TR), $4000 \mathrm{~ms}$; echo time (TE), $70 \mathrm{~ms}$; flip angle, $80^{\circ}$; slice thickness, $4.0 \mathrm{~mm}$; plane resolution, $0.759 \times 0.759 \mathrm{~mm}$; parallel imaging factor, 
16; and matrix, $448 \times$ 448. Afterward, a fat-suppressed T1-weighted three-dimensional sequence of DCE-MRI scans was acquired; this sequence contained one precontrast and five sequentially scanned postcontrast bilateral sagittal image series. The DCE-MRI parameters were as follows: TR, $4.51 \mathrm{~ms}$; TE, $1.61 \mathrm{~ms}$; matrix, 448×448; flip angle, $10^{\circ}$; field of view, $340 \times 340 \mathrm{~mm}$; and in-plane resolution, $0.759 \times 0.759 \mathrm{~mm}$. A bolus of $0.1 \mathrm{mmol} / \mathrm{kg}$ gadobutrol was intravenously injected using an MRI-compatible power injector at a rate of $2 \mathrm{~mL} / \mathrm{s}$. The time between contrast material injection and the start of the first postcontrast acquisition was $60 \mathrm{~s}$. Five consecutive contrast-enhanced image series were then obtained with a temporal resolution of $60 \mathrm{~s}$.

\subsection{Image Processing}

For all cases, image segmentation was performed on the third postcontrast image series because this series usually showed the highest enhancement value during image acquisition and, thus, was usually useful for visual inspection. Radiologists who had 20 years of experience first annotated the centre of the suspicious breast tumour. Using an initial seed from the manually annotated location, a spatial fuzzy C-means (FCM) algorithm automatically segmented a volumetric breast tumour boundary. A Markov random field (MRF)-based approach was subsequently used to refine the initial segmentation, while its parameters were adaptively adjusted using the segmented, contiguous slices (Yang et al., 2014). The ROI covered the whole tumour and was delineated on both the T2W and DCE-MR images of each slice. Because inaccuracies may exist in the automatic segmentation of the tumours, the 
segmentation results were manually examined and corrected by the investigators, with corrections made in less than $5 \%$ of the cases. The sequential DCE-MR images acquired from MRI scans were registered by the same procedure used in a previous study (Yang et al., 2014). Additionally, the radiologists aligned the tumour ROI from DCE-MRI to that of T2WI using the rigid registration tool in Statistical Parametric Mapping 12 (SPM12) (http://www.fil.ion.ucl.ac.uk/spm/software/spm12), which is a common toolbox for image processing, including image registration (Cao et al., 2019).

\subsection{Image Feature Extraction}

For each tumour, image features, including 10 statistical features, 10 morphological features, and 19 texture features obtained based on 3D analysis from the grey-level co-occurrence matrix (GLCM) and 11 texture features calculated from the grey-level size zone matrix (GLSZM), were extracted from the 3D lesion. A detailed description of the image features is provided in Table 2. The statistical feature reflects tumour heterogeneity in terms of the distribution of the voxel intensities inside the 3D lesion. The second-order texture features were calculated using 32 grey levels for both GLCM- and GLSZM-based analysis (Chen et al., 2007; Dong et al., 2018; Haralick et al., 1973). From the DCE-MR images, the texture features and histogram distribution were calculated from the sequential scans of the precontrast series (S0) and from the difference between the intermediate (third) or last (fifth) postcontrast series and the precontrast series; these differences were called S30 and S50, respectively. Morphological features were extracted from S0. A total of 130 image features were 
extracted from DCE-MRI and T2W image scans. Features were normalized by the Z-score normalization method, in which data were transformed into scores with a mean of 0 and a standard deviation of 1 .

Table 2. Image feature description

\begin{tabular}{ll}
\hline Feature class & Feature description \\
\hline $\begin{array}{l}\text { Histogram distribution } \\
(\mathrm{n}=10)\end{array}$ & Mean, Standard Deviation, Kurtosis, Skewness, Maximum, Minimum, \\
& Median, Interquartile range, Range, Entropy \\
\hline Texture GLCM (n=19) & Energy, Contrast, Correlation, Sum of Squares Variance, Homogeneity, \\
& Sum Average, Sum Variance, Sum Entropy, Entropy, Difference Variance, \\
& Difference Entropy, Information Correlation 1, Information Correlation 2, \\
& Autocorrelation, Dissimilarity, Cluster Shade, Cluster Prominence, \\
& Maximum Probability, Inverse Difference \\
\hline Texture & Small Zone Size Emphasis, Large Zone Size Emphasis, Low Grey-Level \\
$(\mathrm{n}=11)$ & Zone Emphasis, High Grey-Level Zone Emphasis, Small Zone Low Grey \\
& Emphasis, Small Zone High Grey Emphasis, Large Zone Low Grey \\
& Emphasis, Large Zone High Grey Emphasis, Grey-Level Nonuniformity, \\
& Zone Size Nonuniformity, Zone Percentage \\
\hline Morphological (n=10) & Volume, Eccentricity, Surface area, Compactness, Maximum Lesion \\
& Radius, Mean Radius of Lesion, Radius of the Sphere, Compactness 1, \\
& Compactness 2, Surface Area/Volume \\
\hline
\end{tabular}

\subsection{Pathological Evaluation}

The histological grade was identified by independent analysis of full-face sections of the breast specimens by two pathologists with more than 15 years of experience who were blinded to the results of the MRI examinations. The histological type of breast cancer was defined according to the World Health Organization (WHO) classification. The tumour histological grade was assessed by the Elston-Ellis System (Elston and Ellis, 1991).

\subsection{CCA-based Feature Fusion}

Mathematically, CCA aims to find a set of basis vector pairs that maximize the 
correlation between two different sample sets obtained from two types of images with the same information source. We define $\left\{x_{i}\right\}_{i=1}^{n} \subset R^{p}$ and $\left\{x_{i}\right\}_{i=1}^{n} \subset R^{q}$ as features from different types of images (i.e., DCE-MRI and T2WI) of the same subject, where $p$ and $q$ denote the number of features from these images and $n$ denotes the number of patients. CCA was used to identify the two projection directions, $w_{x} \in R^{p}$ and $w_{y} \in$ $R^{q}$, that maximize the correlation $r$ between $w_{x}^{T} X$ and $w_{y}^{T} Y$, where $r$ is defined as follows:

$$
r=\max \frac{w_{x}^{T} C_{x y} w_{y}}{\sqrt{\left(w_{x}^{T} C_{x x} w_{x}\right)\left(w_{y}^{T} C_{y y} w_{y}\right)}}
$$

where $C_{x y}=X Y^{T}$ denotes the between-set covariance matrix and $C_{x x}=X X^{T}$ and $C_{y y}=Y Y^{T}$ denote the within-set covariance matrices.

When the covariance matrices $C_{x x}$ and $C_{y y}$ are nonsingular, the CCA solution can be obtained by solving the following generalized eigen-problem. Let $w_{x}=$ $\left[w_{x 1}, w_{x 2}, \ldots, w_{x d}\right]$ and $w_{y}=\left[w_{x 1}, w_{x 2}, \ldots, w_{x d}\right]$ denote two projection direction matrices, where the vector pairs $\left(w_{x i}, w_{y i}\right)_{i=1}^{d}$ correspond to the first $d$ largest generalized eigenvalues. For any sample pair $(x, y)$ from the two parametric images, we can obtain the fused feature through the following equation:

$$
\mathrm{z}=\frac{1}{2}\left(w_{X}^{T} x+w_{X}^{T} y\right)
$$

In our case, the dimensionality of the features is less than the sample size; hence, the within-set covariance matrices $C_{x x}$ and $C_{y y}$ are not singular.

\subsection{Feature Selection and Classification}

In the development cohort, a 10-fold cross validation (CV) strategy was used to perform feature selection and model parameter tuning. A support vector machine with 
the recursive feature elimination (SVM-RFE) algorithm was performed to identify an optimal feature subset with the best discriminative power (Guyon et al., 2002). The goal of SVM-RFE is to determine a ranking list for all $M$ features based on a backward sequential selection strategy, in which the weight of each feature is obtained and the one with the smallest weight is iteratively eliminated. Therefore, top-ranked features are those that remained through the largest number of iterations. Using the rank order, SVM classifiers were constructed with a varying number of top-ranked features (top $1,2,3, \ldots$ up to $M / 2$ ). The optimal feature subset is determined when the first $N$ features in the feature list achieve the highest classification performance in terms of AUC value.

A grid search approach was performed in the training process to select the optimal values of two parameters, $C$ (penalty parameter) and $\sigma$ (kernel parameter of RBF), of the SVM classifier. The optimal values of these two parameters are determined when they yield the largest classification accuracy based on 10-fold CV. The predictive model with the optimal feature sets and the tuned model parameters was built using all of the 111 training samples, and this model was tested on the validation cohort.

\subsection{Statistical Analysis}

The individual and collective performances of the image features were examined by the SVM classifier to assess the area under the receiver operating characteristic (ROC) curve (AUC). Before application of the feature fusion, we removed redundant features with high similarity to reduce the impact of data co-linearity on the classification model. More specifically, the features were removed when the Pearson coefficient 
correlations (PCCs) with other features were greater than 0.9. For each of the two features with high similarity, the feature with higher average correlations with the other features was removed. Thereafter, feature fusion was applied between T2W images and each DCE-MRI series. A corrected p-value less than 0.05 was determined to be statistically significant. All statistical analyses were performed using MATLAB software (2015a MathWorks Inc).

\section{RESULTS}

\subsection{Performance of Individual Imaging Features in Histological Grade}

\section{Prediction}

The performance of individual features (in terms of AUC) from DCE-MRI and T2W images was examined in terms of their ability to determine the tumour histological grade in the validation set (Table 3 and Figure 2). From the figure, the morphological features showed the most predictive power, texture features had intermediate performance and statistical features had the lowest discriminative ability for histological grade prediction. A detailed list of the top 30 individual feature performances in terms of AUC values is shown in Table 3. From this table, morphological features, such as the radius of the sphere, volume, surface area, and compactness 1 , showed the best prediction performances, with mean AUC values of $0.771,0.763,0.755$ and 0.754 , respectively. Higher values of these features are associated with higher tumour grade. We observed relatively high performance for a texture feature of information correlation 2 from the S0, S30, and S50 DCE-MRI and T2W images, with AUC values of $0.745,0.753,0.767$ and 0.754 , respectively. 
Table 3. Performance of individual imaging features in association with pathological grade.

\begin{tabular}{|c|c|c|c|c|c|}
\hline \multirow{2}{*}{ Feature } & \multicolumn{5}{|c|}{ AUC } \\
\hline & S0 & S30 & S50 & T2WI & Mean \\
\hline Radius of the Sphere & 0.760 & 1 & 1 & 0.783 & 0.771 \\
\hline Volume & 0.753 & 1 & 1 & 0.773 & 0.763 \\
\hline Information Correlation 2 & 0.745 & 0.753 & 0.767 & 0.754 & 0.755 \\
\hline Surface Area & 0.746 & 1 & 1 & 0.763 & 0.755 \\
\hline Compactness 1 & 0.749 & 1 & 1 & 0.759 & 0.754 \\
\hline Information Correlation 1 & 0.702 & 0.759 & 0.789 & 0.747 & 0.749 \\
\hline Eccentricity & 0.720 & 1 & 1 & 0.737 & 0.729 \\
\hline Large Zone High Grey Emphasis & 0.698 & 0.708 & 0.630 & 0.734 & 0.693 \\
\hline Grey-Level Nonuniformity & 0.647 & 0.686 & 0.742 & 0.686 & 0.690 \\
\hline Zone Percentage & 0.729 & 0.615 & 0.647 & 0.763 & 0.688 \\
\hline Zone Size Nonuniformity & 0.740 & 0.635 & & 0.766 & 0.688 \\
\hline Surface Area/Volume & 0.766 & 1 & & 0.605 & 0.686 \\
\hline Small Zone Size Emphasis & 0.659 & 0.686 & 0.733 & 0.656 & 0.684 \\
\hline Compactness 2 & 0.624 & & & 0.728 & 0.676 \\
\hline Dissimilarity & 0.632 & & 0.667 & 0.656 & 0.655 \\
\hline Large Zone Low Grey Emphasis & 0.740 & 0.535 & 0.539 & 0.801 & 0.654 \\
\hline Homogeneity & 0.721 & 0.712 & 0.622 & 0.533 & 0.647 \\
\hline Contrast & 0.609 & 0.689 & 0.664 & 0.598 & 0.640 \\
\hline Maximum Lesion Radius & & 1 & 1 & 0.721 & 0.638 \\
\hline Entropy & 0.483 & 0.798 & 0.672 & 0.568 & 0.630 \\
\hline Inverse Difference & 0.646 & 0.508 & 0.695 & 0.570 & 0.605 \\
\hline Large Zone Size Emphasis & 0.694 & 0.344 & 0.667 & 0.714 & 0.604 \\
\hline Small Zone Low Grey Emp & 0.578 & 0.633 & 0.680 & 0.520 & 0.603 \\
\hline Cluster Prominence & 0.590 & 0.551 & 0.703 & 0.530 & 0.593 \\
\hline Maximum & 0.542 & 0.501 & 0.616 & 0.678 & 0.584 \\
\hline Energy & 0.504 & 0.654 & 0.633 & 0.545 & 0.584 \\
\hline Difference Entropy & 0.630 & 0.392 & 0.527 & 0.699 & 0.562 \\
\hline Mean & 0.581 & 0.427 & 0.648 & 0.582 & 0.560 \\
\hline High Grey-Level Zone Emphasis & 0.648 & 0.560 & 0.671 & 0.353 & 0.558 \\
\hline Difference Variance & 0.538 & 0.561 & 0.549 & 0.579 & 0.557 \\
\hline
\end{tabular}

S0, S30 and S50 represent the precontrast series and the differences (subtraction) between the intermediate (the third) and last (the fifth) postcontrast series and the precontrast series, respectively. For DCE-MRI, morphological features were extracted from the precontrast series $(\mathrm{S} 0)$. 

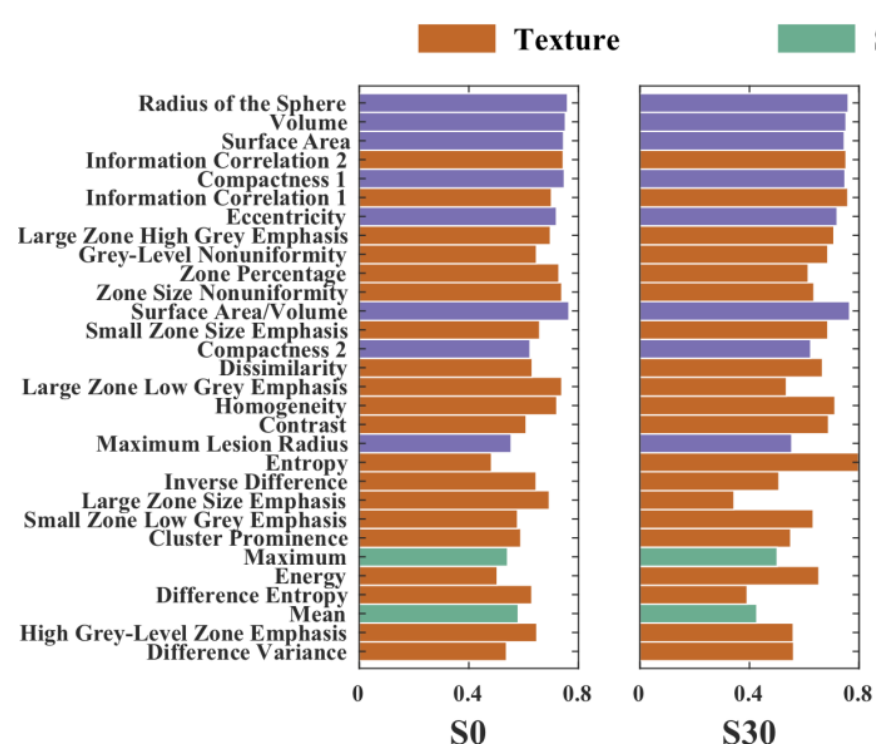

\section{Statistics}

Morphologic

Figure 2. The performance of the top 30 features from the DCE-MRI series and T2W imaging in terms of AUC values.

Figure 3 shows examples of this image feature in multiparametric images for discriminating the histological grade. Patients with an overall lower value for this feature were observed to have a higher grade from the DCE-MRI and T2W image series, and this trend was more obvious in the S2 and S3 images than in the S0 and T2W images. 

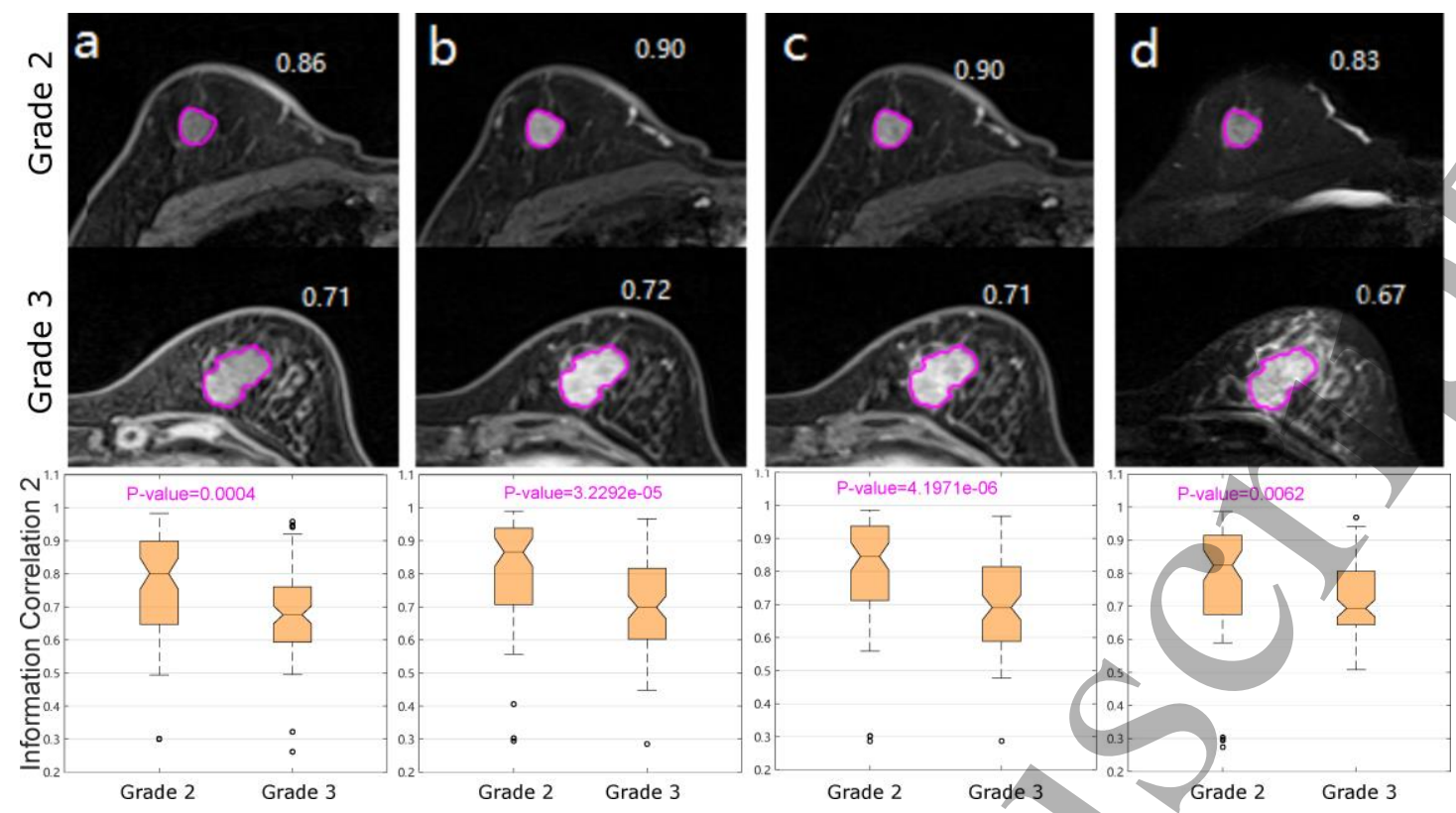

Figure 3. Examples of the information correlation 2 feature in two patients with grade 2 (the first row) and grade 3 (the second row) tumours. The third row shows the distribution of this feature in the low/intermediate-grade and high-grade groups. A) Precontrast series of DCE-MRI of grade 2 and grade 3 tumours with feature values of 0.86 and 0.71 , respectively. Image feature examples and distributions are shown in the B) third postcontrast, C) fifth postcontrast, and D) T2W images.

\subsection{Predictive Model Based on DCE-MRI and T2W Images for Training and}

\section{Testing}

The collective performance of the image features obtained from the T2W, S0, S30 and S50 images was assessed, and the predictive models showed AUC values of 0.750 , 0.749, 0.788 and 0.769 , respectively, for the development cohort $(n=111)$. Among those images, S30 and S50 showed higher performance in terms of AUC values than T2W and S0.

The established predictive model using the optimal feature set and tuned model parameters was established using all the samples in the development cohort and was 
tested with the validation cohort $(n=56)$. The performance of the predictive models based on the T2W, S0, S30 and S50 images showed AUC values of 0.715, 0.704, 0.744 and 0.716 , respectively (Figure 4). Similar to the results for the development cohort, S30 and S50 showed higher performances than T2W and S0 in terms of AUC values.

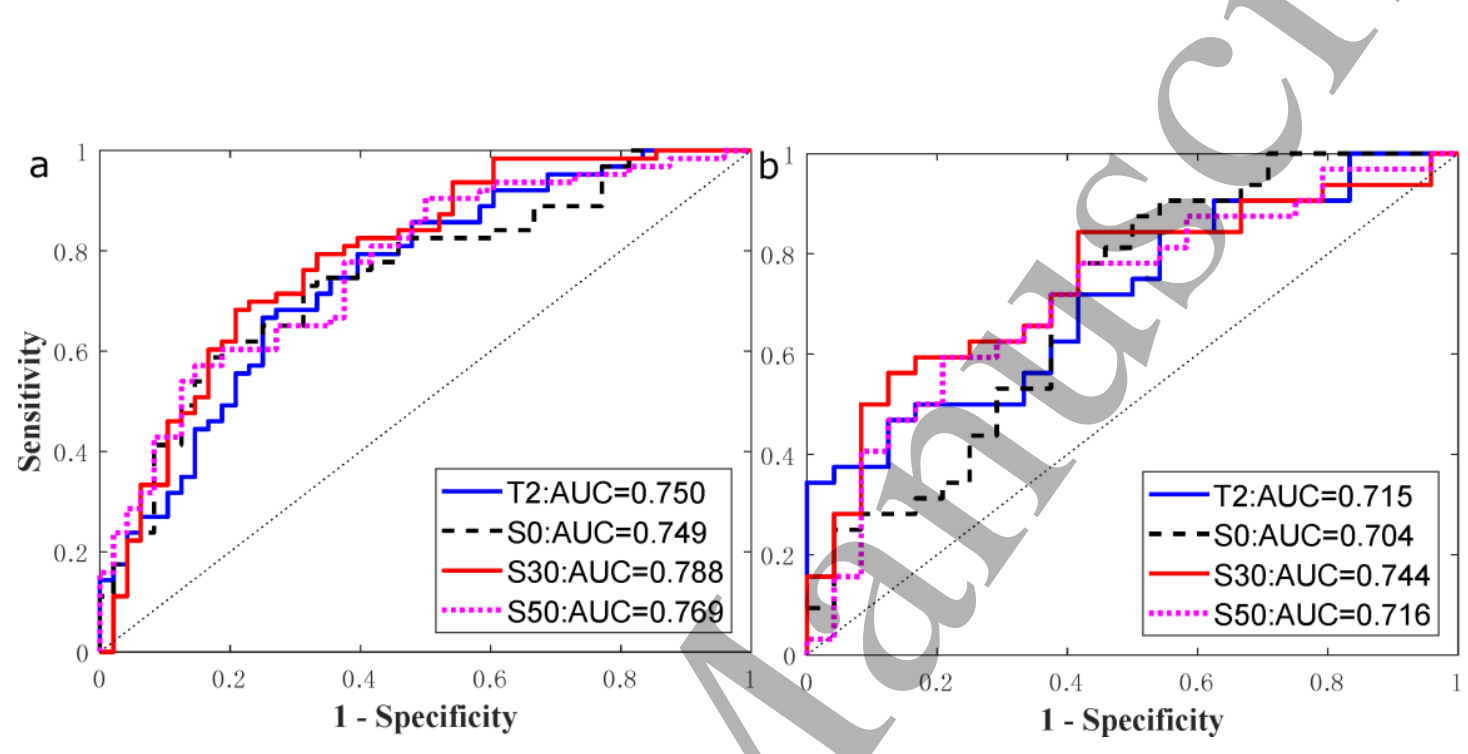

Figure 4. ROC plots of image features from the S0, S30, and S50 images in the (a) development and (b) validation cohort.

\subsection{Predictive Models Using Fusion Methods Based on Multiparametric Image \\ Features}

Information from DCE-MRI and $\mathrm{T} 2 \mathrm{~W}$ images was further integrated by feature concatenation, classifier fusion or CCA-based methods for better prediction. As shown in Table 4, the classifier based on prediction score fusion and concatenation of features from DCE-MRI and T2W images showed slightly better performance than the classifier based on a single image series. 
Table 4. Classifier performance (in terms of AUCs) in image series of DCE-MRI and T2WI

\begin{tabular}{|c|c|c|c|c|}
\hline \multirow{2}{*}{ Image series } & \multirow{2}{*}{ Original } & \multirow{2}{*}{ Classifier Fusion } & \multicolumn{2}{|c|}{ Feature Fusion } \\
\hline & & & Concatenation & CCA \\
\hline \multicolumn{5}{|c|}{ AUC in development cohort } \\
\hline T2WI & $0.750 \pm 0.047$ & N/A & N/A & N/A \\
\hline So & $0.749 \pm 0.047$ & $0.759 \pm 0.046$ & $0.777 \pm 0.045$ & $0.778 \pm 0.047$ \\
\hline $\mathrm{S} 30$ & $0.788 \pm 0.045$ & $0.783 \pm 0.045$ & $0.789 \pm 0.046$ & $0.822 \pm 0.040$ \\
\hline S50 & $0.769 \pm 0.045$ & $0.776 \pm 0.044$ & $0.788 \pm 0.045$ & $0.805 \pm 0.045$ \\
\hline \multicolumn{5}{|c|}{ AUC in validation cohort } \\
\hline T2WI & $0.715 \pm 0.068$ & N/A & N/A & \\
\hline S0 & $0.704 \pm 0.073$ & $0.720 \pm 0.069$ & $0.737 \pm 0.067$ & $0.751 \pm 0.065$ \\
\hline $\mathrm{S} 30$ & $0.744 \pm 0.067$ & $0.750 \pm 0.065$ & $0.754 \pm 0.068$ & $0.803 \pm 0.060$ \\
\hline $\mathrm{S} 50$ & $0.716 \pm 0.071$ & $0.754 \pm 0.065$ & $0.758 \pm 0.064$ & $0.794 \pm 0.060$ \\
\hline
\end{tabular}

$\overline{\mathrm{CCA}}=$ canonical correlation analysis; DCE-MRI = Dynamic contrast-enhanced magnetic resonance imaging; T2WI = T2-weighted imaging; NA = not applicable.

S0, S30 and S50 represent the precontrast series and the difference (subtraction) between the intermediate (third) or the last (fifth) postcontrast series and the precontrast series, respectively.

We compared the effectiveness of CCA-based feature fusion and the other fusion methods (i.e., prediction score fusion and concatenation). As described, the S30-based image series generated a prediction performance with an AUC of 0.788 in the development cohort and 0.744 in the validation cohort. After the fusion of features from S30 and T2W images using CCA, the AUCs increased to 0.822 in the development cohort and 0.803 in the validation cohort. Moreover, the performance of this predictive model was also higher than that based on either the prediction score or concatenation-based methods (Table 4 and Figure 5). Regarding the S50 subtraction image, the predictive model generated an AUC of $0.716 \pm 0.071$, which was improved to an AUC of 0.794 by fusion with the T2WI features using CCA. The method of fusing features between DCE-MRI and T2W images using CCA achieved better performance than the concatenation-based feature fusion and classifier fusion 
methods.

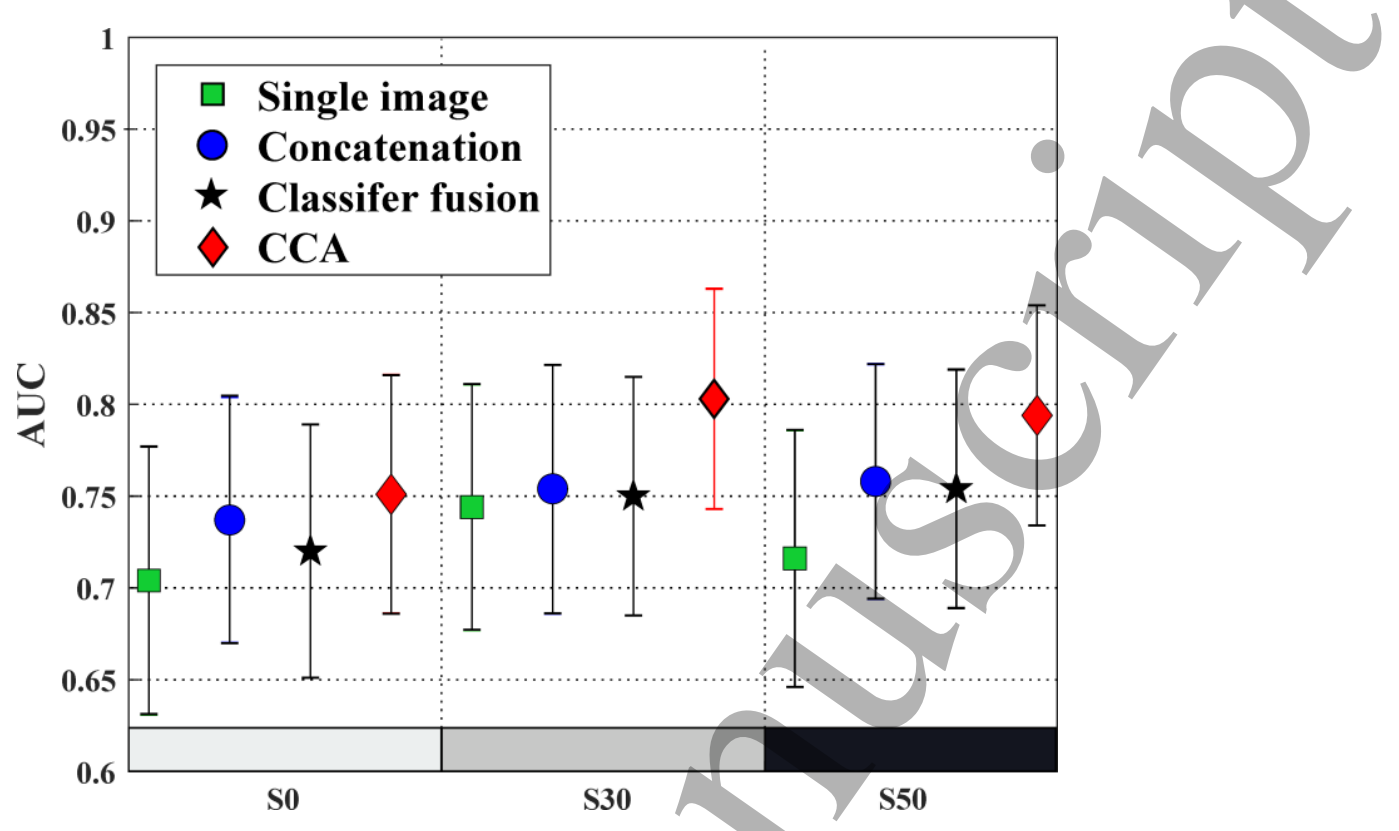

Figure 5. Performance in terms of AUC values for different image series by applying the following methods to features: single MRI series, classifier prediction score fusion, concatenation of features and feature fusion by CCA.

\subsection{Model Performances Using Image Features in Combined DCE-MRI Series}

We also established a predictive model by concatenating features from three images (i.e., S0, S30 and S50). Combined features from S0 and S20 (i.e., S0+S20), S0 and S30 (i.e., S0+S30), S30 and S50 (i.e., S3+S50) and S0, S30, S50 (i.e., S0+S30+S50) were evaluated for prediction (Figure 6 and Table 5). The predictive model using combined features from S0 and S30 showed higher performance than the other combinations; the AUC value was 0.771 in the development cohort and 0.725 in the validation cohort. 

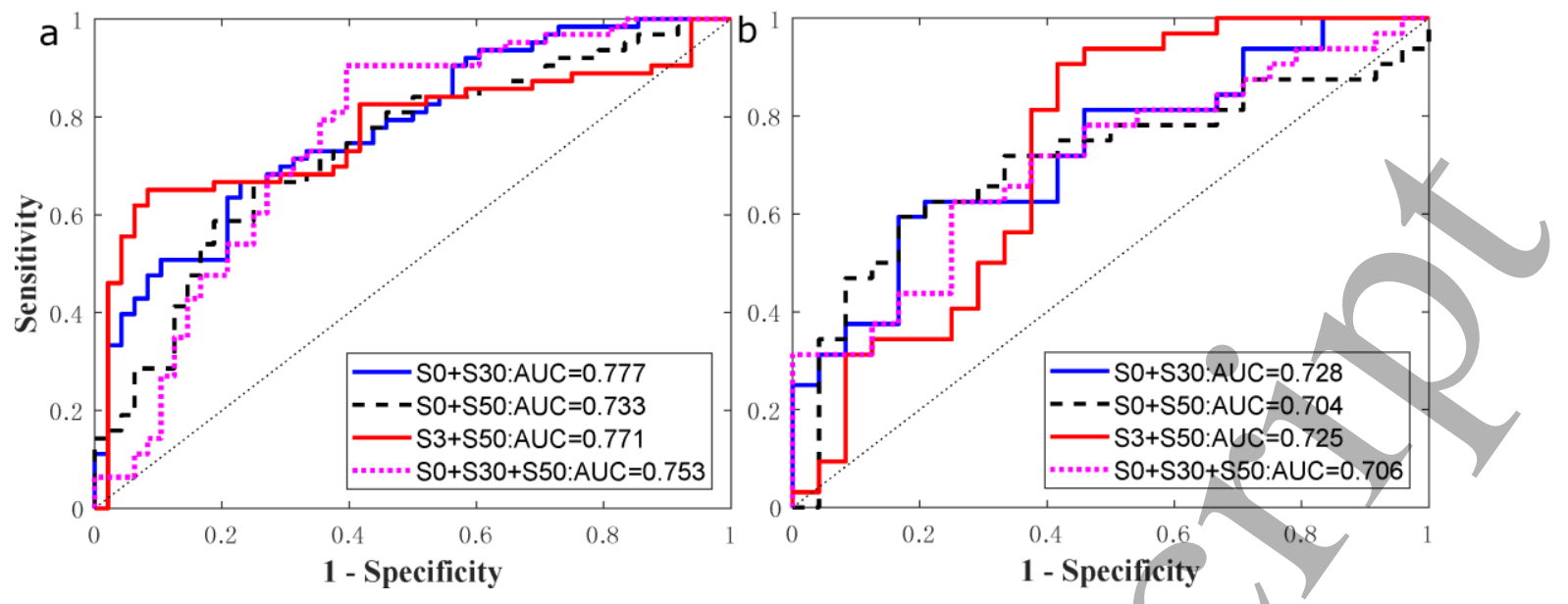

Figure 6. ROC plots of image features from the S0, S30, and S50 images in the (a) development and (b) validation cohort.

Predictive models generated by the fusion of T2WI and combined DCE-MRI series were implemented using the classifier fusion and feature fusion methods. Table 5 shows the classifier performance using the combined DCE-MRI series and the fusion of T2WI. After the fusion of features from combined S30 and S50 subtraction images and T2W images using CCA, model performance was increased to 0.808 in the development cohort and 0.785 in the validation cohort. Moreover, the performance of the combination of all three DCE-MRI series was improved by CCA-based fusion with T2WI and was greater than that of the other fusion methods. The results showed that although combining features via concatenation-based feature fusion could improve prediction performance, higher accuracies were obtained by the CCA-based fusion method. 
Table 5. Classifier performance using the combined DCE-MRI series and the fusion with T2WI

\begin{tabular}{|c|c|c|c|c|}
\hline \multirow{2}{*}{ Image series } & \multirow{2}{*}{ Original } & \multirow{2}{*}{ Classifier Fusion } & \multicolumn{2}{|c|}{ Feature Fusion } \\
\hline & & & Concatenation & CCA \\
\hline \multicolumn{5}{|c|}{ AUC in development cohort } \\
\hline $\mathrm{S} 0+\mathrm{S} 30$ & $0.777 \pm 0.044$ & $0.778 \pm 0.044$ & $0.769 \pm 0.050$ & $0.787 \pm 0.046$ \\
\hline $\mathrm{S} 0+\mathrm{S} 50$ & $0.733 \pm 0.048$ & $0.756 \pm 0.046$ & $0.769 \pm 0.048$ & $0.786 \pm 0.048$ \\
\hline $\mathrm{S} 3+\mathrm{S} 50$ & $0.771 \pm 0.046$ & $0.759 \pm 0.045$ & $0.780 \pm 0.048$ & $0.808 \pm 0.047$ \\
\hline $\mathrm{S} 0+\mathrm{S} 30+\mathrm{S} 50$ & $0.753 \pm 0.049$ & $0.782 \pm 0.045$ & $0.790 \pm 0.045$ & $0.799 \pm 0.045$ \\
\hline
\end{tabular}

\begin{tabular}{|c|c|c|c|c|}
\hline \multicolumn{5}{|c|}{ AUC in validation cohort } \\
\hline $\mathrm{S} 0+\mathrm{S} 30$ & $0.728 \pm 0.068$ & $0.731 \pm 0.067$ & $0.741 \pm 0.070$ & $0.750 \pm 0.066$ \\
\hline $\mathrm{S} 0+\mathrm{S} 50$ & $0.704 \pm 0.072$ & $0.724 \pm 0.067$ & $0.729 \pm 0.068$ & $0.764 \pm 0.067$ \\
\hline $\mathrm{S} 3+\mathrm{S} 50$ & $0.725 \pm 0.074$ & $0.745 \pm 0.067$ & $0.751 \pm 0.069$ & $0.785 \pm 0.061$ \\
\hline $\mathrm{S} 0+\mathrm{S} 30+\mathrm{S} 50$ & $0.706 \pm 0.070$ & $0.738 \pm 0.066$ & $0.749 \pm 0.068$ & $0.762 \pm 0.063$ \\
\hline
\end{tabular}

$\mathrm{CCA}=$ canonical correlation analysis; DCE-MRI = dynamic contrast-enhanced magnetic resonance imaging; T2WI = T2-weighted imaging;

S0, S30 and S50 represent the precontrast series and the difference (subtraction) between the intermediate (third) or the last (fifth) postcontrast series and the precontrast series, respectively.

DCE-MRI series are incorporated (e.g., S30+S50 indicates that features from these images are concatenated).

\subsection{Optimal Feature Subset Analysis}

Based on the features extracted from tumour ROIs on DCE-MRI, an optimal subset was determined by using SVM-RFE. For the S30 image series, the results show that the first four features achieved the highest AUC (Figure 7). Among the four features, two were morphological features (i.e., maximum lesion radius, surface area/volume), one was a statistical feature (i.e., minimum) and one was a texture feature (i.e., zone percentage). In the predictive model, the optimal parameters for the SVM classifier were $C=0.0625$ and $\sigma=2$. 


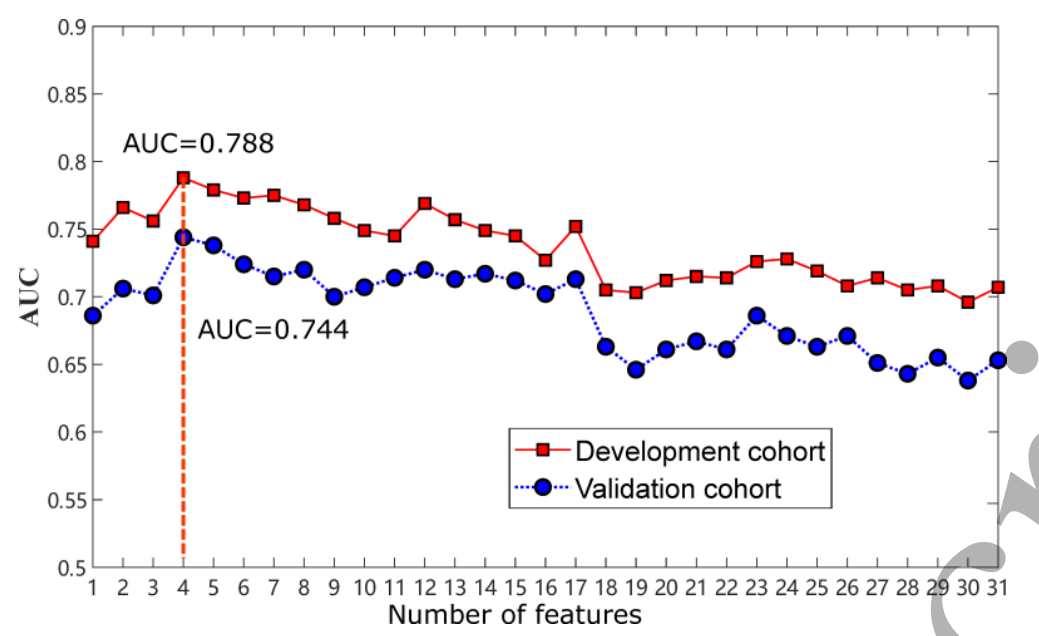

Figure 7. Performance of the S30-based predictive model with a varied number of input features.

In this study, the original features identified from two types of images were fused by linear transformation; hence, the averaged canonical yariables in the predictive model represented common or complementary information between these images. We evaluated whether the varied number of features could affect the performance of predictive models that combined S30 and T2WI features using CCA, and the results are shown in Figure 8. In this model, CCA generated 29 averaged canonical variables, which were ranked by their correlation coefficients. Then, the optimal subset was determined using SVM-RFE. The highest performance was achieved using 24 features (Figure 8). The optimal parameters for SVM were $\mathrm{C}=0.5$ and $\sigma=0.0078$. The most important variable in SVM-RFE showed the highest correlation coefficient $(r=1)$ between the paired canonical variables. However, the other features with lower correlations also showed a relatively high selection frequency. Notably, both features with high correlation between paired canonical variables and features with relatively low correlation exhibited high importance in the predictive model. 


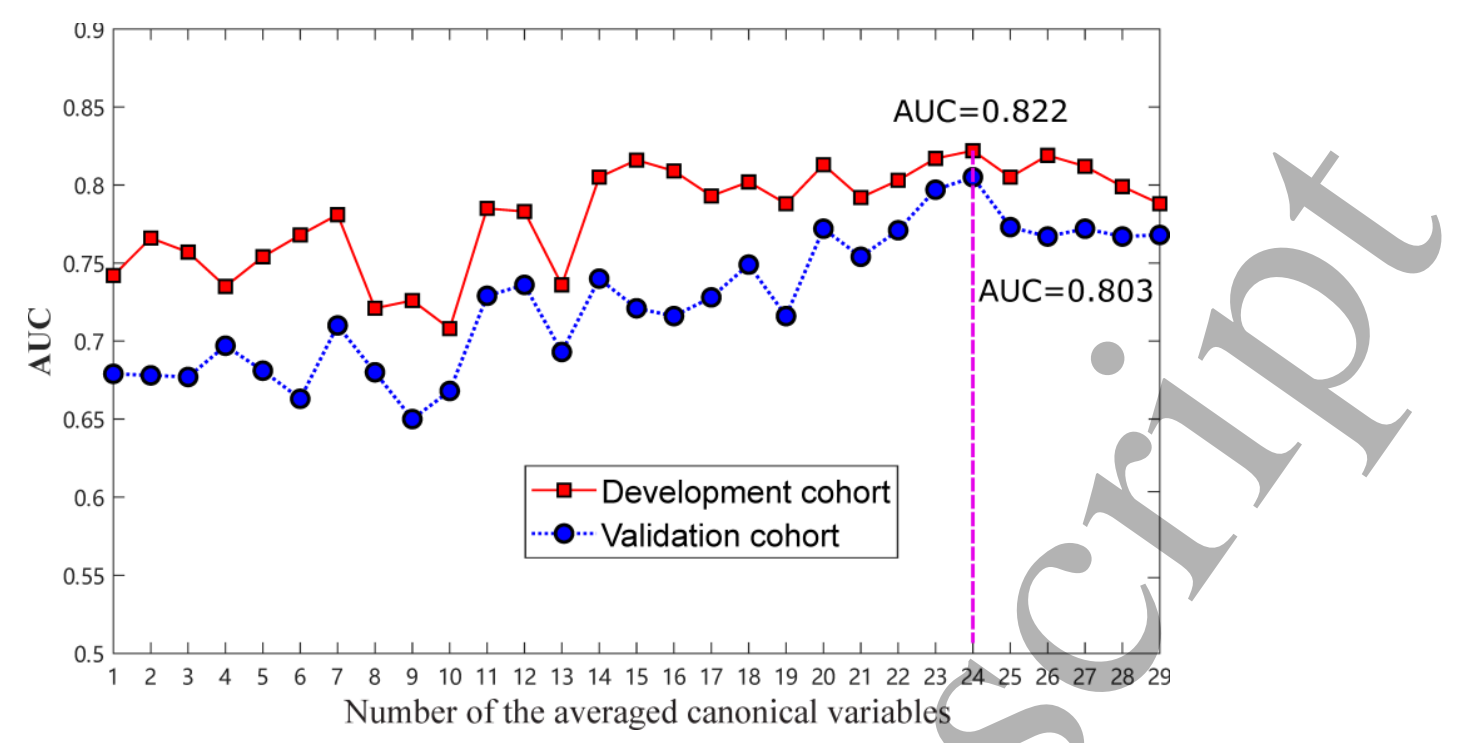

Figure 8. Performance of the predictive model using the CCA-based feature fusion method with a varied number of averaged canonical variables.

\section{DISCUSSION}

In this study, we performed radiomic analysis based on a multiparametric MRI series to predict the histological grade of IDC. Our work represents a preliminary study of radiomic feature fusion analysis using heterogeneous sources of tumour images. Our results demonstrate that compared with analysis based on a single image source, a machine learning model with a feature fusion method that optimizes correlations between features from multiple sources of MRI images obtained from breast tumours has better predictive power for discriminating the histological grade in IDC.

Previous studies performed radiomic analysis to assess the association between histopathological information, including breast cancer subtypes (Grimm, 2016; Li et al., 2016; Mazurowski et al., 2014) and response to neoadjuvant chemotherapy (Braman et al., 2017; Fan et al., 2017b). An early study found that larger tumour size 
is associated with higher grade in breast cancer, which is somewhat consistent with our finding that a higher value of the radius of the sphere or volume feature is correlated with a higher tumour grade (Jeh et al., 2011). A recent study identified heterogeneity-related texture features based on DCE-MRI for predicting breast cancer proliferation indicators (Fan et al., 2018). Parekh et al. identified multiparamẹtric tumour images for the discrimination of benign and malignant tumours (Parekh and Jacobs, 2017). Dong et al. predicted SLN metastasis in breast cancer patients using radiomic features from T2-weighted fat suppression (T2-FS) and diffusion-weighted MRI (DWI) (Dong et al., 2018). However, these studies did not evaluate the relationship between these types of images and hence did not provide information on whether the combination of these images could improve the prediction accuracy. In this study, we identified DCE-MRI and T2W radiomic features from the same tumour to examine the correlation between heterogeneous sources of tumour images.

We observed that CCA-based feature fusion improved the prediction of the pathological status compared to that obtained from the single image series, the concatenation-based feature fusion method and the decision-level fusion method. Moreover, CCA could retain as much of the identity information as possible and further reduce the redundant information within the features to obtain a more discriminative set of features (Haghighat et al., 2016). In our tests, the top-ranking feature in SVM-RFE was the feature with the highest correlation coefficient $(r=1)$ between the paired canonical variables. Therefore, the exploitation of similar and complementary tumour representations by linear transformation of the image features 
from different parametric images can be used to identify the most similar pattern that may be useful for the prediction and can overcome the limitations of heterogeneous MRI assessments. Despite the noise in each individual source of tumour examination, our experiments show that the combination of these sources in a feature fusion approach improves the prediction.

In the context of artificial intelligence (AI), convolutional neural network $(\mathrm{CNN})$ methods are capable of learning features from large imaging datasets, which has tremendous potential in radiology applications (Akay and Hess, 2019; Shin et al., 2016). A recent study compared $\mathrm{CNN}$ and radiomics-based methods for the classification of lesions as benign or malignant in multiparametric breast MRI (Truhn et al., 2019). In this study, the diagnostic performance of CNN was superior to that of radiomic analysis; the performance of CNN was improved by using more training data, whereas that of the radiomic analysis-based method was not improved. CNN methods could enhance the performance of feature fusion by concatenating the deep features in the last hidden layer from different inputs of images, while decision-level fusion is performed by combining the decisions made by each predictor (Xie et al., 2018). Therefore, further deep feature fusion methods that capture linear/nonlinear correlations between different parametric images could be used to achieve more accurate predictions.

Despite some significant findings, this study has several limitations. For example, the size of the dataset is small, and the robustness of our predictive models needs to be further validated in future studies using a large independent dataset with different 
imaging protocols. Furthermore, we used a state-of-the-art feature fusion method to measure the similarity and difference between two types of images with linear transformation of the image features. Thus, additional studies of feature fusion techniques (e.g., deep $\mathrm{CNN}$ ) with nonlinear transformations are also needed to refine this study. We performed spatial texture analysis of tumour MR images, whereas spatiotemporal DCE-MRI texture analysis should also be conducted to examine the effectiveness (Wang et al., 2015a) (Fave et al., 2017). In our experiments, tumour ROIs were first segmented by FCM and MRF methods and were further manually corrected by radiologists if needed, although such manual correction may introduce bias caused by user variability. Further reliable, validated image segmentation methods should be conducted and compared to evaluate the robustness of radiomic analysis. Finally, it would be of interest to perform feature fusion using other parametric images, such as DWI, for more accurate prediction (Martincich et al., 2012; Razek et al., 2010).

In conclusion, texture biomarkers are a quantitative measurement of tumour heterogeneity in multiparametric MRI scans of IDC that can be used to predict tumour histological grade. We have explored a novel approach using feature fusion methods based on both anatomical and functional MR images to better quantify the correlation between different parametric images from the same information source. Predictive models based on quantitative radiomic features could potentially be useful for prognosis and treatment management in breast cancer.

\section{Acknowledgements}


This work has received funding from the National Natural Science Foundation of China (61871428, 61731008, U1809205 and 61401131), the Natural Science Foundation of Zhejiang Province of China (LJ19H180001), and the King Abdullah University of Science and Technology (KAUST) Office of Sponsored Research (OSR) under Award No. URF/1/3450-01.

\section{References}

Abraham, B., and Merola, G. (2005). Dimensionality reduction approach to multivariate prediction. Comput Stat Data An 48, 5-16.

Akay, A., and Hess, H. (2019). Deep Learning: Current and Emerging Applications in Medicine and Technology. IEEE journal of biomedical and health informatics 23, 906-920.

Bhooshan, N., Giger, M., Lan, L., Li, H., Marquez, A., Shimauchi, A., and Newstead, G.M. (2011). Combined use of T2-weighted MRI and T1-weighted dynamic contrast-enhanced MRI in the automated analysis of breast lesions. Magnetic resonance in medicine 66, 555-564.

Braman, N.M., Etesami, M., Prasanna, P., Dubchuk, C., Gilmore, H., Tiwari, P., Plecha, D., and Madabhushi, A. (2017). Intratumoral and peritumoral radiomics for the pretreatment prediction of pathological complete response to neoadjuvant chemotherapy based on breast DCE-MRI. Breast cancer research : BCR 19, 57.

Burnside, E.S., Drukker, K., Li, H., Bonaccio, E., Zuley, M., Ganott, M., Net, J.M., Sutton, E.J., Brandt, K.R., Whitman, G.J., et al. (2016). Using computer-extracted image phenotypes from tumors on breast magnetic resonance imaging to predict breast cancer pathologic stage. Cancer 122, 748-757.

Cao, R., Bajgiran, A.M., Mirak, S.A., Shakeri, S., Zhong, X., Enzmann, D., Raman, S., and Sung, K. (2019). Joint Prostate Cancer Detection and Gleason Score Prediction in mp-MRI via FocalNet. IEEE transactions on medical imaging.

Chang, Y.W., Kwon, K.H., Choi, D.L., Lee, D.W., Lee, M.H., Lee, H.K., Yang, S.B., Kim, Y., and Seo, D.Y. (2009). Magnetic resonance imaging of breast cancer and correlation with prognostic factors. Acta Radiol 50, 990-998.

Chen, W., Giger, M.L., Li, H., Bick, U., and Newstead, G.M. (2007). Volumetric texture analysis of breast lesions on contrast-enhanced magnetic resonance images. Magnetic resonance in medicine 58, 562-571.

Dong, Y., Feng, Q., Yang, W., Lu, Z., Deng, C., Zhang, L., Lian, Z., Liu, J., Luo, X., Pei, S., et al. (2018). Preoperative prediction of sentinel lymph node metastasis in breast cancer based on radiomics of T2-weighted fat-suppression and diffusion-weighted MRI. European radiology 28, 582-591.

Ehinger, A., Malmstrom, P., Bendahl, P.O., Elston, C.W., Falck, A.K., Forsare, C., Grabau, D., Ryden, L., Stal, O., and Ferno, M. (2017). Histological grade provides significant prognostic information in addition to breast cancer subtypes defined according to St Gallen 2013. Acta Oncol 56, 68-74.

Ellis, M.J., Tao, Y., Luo, J., A'Hern, R., Evans, D.B., Bhatnagar, A.S., Chaudri Ross, H.A., von Kameke, A., Miller, W.R., Smith, I., et al. (2008). Outcome prediction for estrogen receptor-positive breast cancer based on postneoadjuvant endocrine therapy tumor characteristics. Journal of the National Cancer 
Institute 100, 1380-1388.

Elston, C.W., and Ellis, I.O. (1991). Pathological prognostic factors in breast cancer. I. The value of histological grade in breast cancer: experience from a large study with long-term follow-up. Histopathology 19, 403-410.

Engstrom, M.J., Opdahl, S., Vatten, L.J., Haugen, O.A., and Bofin, A.M. (2015). Invasive lobular breast cancer: the prognostic impact of histopathological grade, E-cadherin and molecular subtypes. Histopathology 66, 409-419.

Fan, M., Cheng, H., Zhang, P., Gao, X., Zhang, J., Shao, G., and Li, L. (2018). DCE-MRI texture analysis with tumor subregion partitioning for predicting Ki-67 status of estrogen receptor-positive breast cancers. Journal of magnetic resonance imaging : JMRI 48, 237-247.

Fan, M., Li, H., Wang, S., Zheng, B., Zhang, J., and Li, L. (2017a). Radiomic analysis reveals DCE-MRI features for prediction of molecular subtypes of breast cancer. PloS one 12, e0171683.

Fan, M., Wu, G., Cheng, H., Zhang, J., Shao, G., and Li, L. (2017b). Radiomic analysis of DCE-MRI for prediction of response to neoadjuvant chemotherapy in breast cancer patients. European journal of radiology 94, 140-147.

Fave, X., Zhang, L., Yang, J., Mackin, D., Balter, P., Gomez, D., Followill, D., Jones, A.K., Stingo, F., Liao, Z., et al. (2017). Delta-radiomics features for the prediction of patient outcomes in non-small cell lung cancer. Scientific reports 7,588 .

Gallego-Ortiz, C., and Martel, A.L. (2017). Using quantitative features extracted from T2-weighted MRI to improve breast MRI computer-aided diagnosis (CAD). PloS one 12, e0187501.

Grimm, L.J. (2016). Breast MRI radiogenomics: Current status and research implications. Journal of magnetic resonance imaging : JMRI 43, 1269-1278.

Guyon, I., Weston, J., Barnhill, S., and Vapnik, V. (2002). Gene Selection for Cancer Classification using Support Vector Machines. Mach Learn 46, 389-422.

Haghighat, M., Abdel-Mottaleb, M., and Alhalabi, W. (2016). Discriminant Correlation Analysis: Real-Time Feature Level Fusion for Multimodal Biometric Recognition. IEEE T Inf Foren Sec 11, 1984-1996.

Haralick, R.M., Shanmugam, K., and Dinstein, I. (1973). Textural Features for Image Classification. IEEE Transactions on Systems, Man, and Cybernetics SMC-3, 610-621.

Jeh, S.K., Kim, S.H., Kim, H.S., Kang, B.J., Jeong, S.H., Yim, H.W., and Song, B.J. (2011). Correlation of the apparent diffusion coefficient value and dynamic magnetic resonance imaging findings with prognostic factors in invasive ductal carcinoma. Journal of magnetic resonance imaging : JMRI 33, 102-109.

Kuhl, C.K., Klaschik, S., Mielcarek, P., Gieseke, J., Wardelmann, E., and Schild, H.H. (1999). Do T2-weighted pulse sequences help with the differential diagnosis of enhancing lesions in dynamic breast MRI? Journal of magnetic resonance imaging : JMRI 9, 187-196.

Li, H., Zhu, Y., Burnside, E.S., Hưang, E., Drukker, K., Hoadley, K.A., Fan, C., Conzen, S.D., Zuley, M., Net, J.M., et al. (2016). Quantitative MRI radiomics in the prediction of molecular classifications of breast cancer subtypes in the TCGA/TCIA data set. NPJ breast cancer 2.

Li, L., Wang, K., Sun, X., Sun, Y., Zhang, G., and Shen, B. (2015). Parameters of dynamic contrast-enhanced MRI as imaging markers for angiogenesis and proliferation in human breast cancer. Medical science monitor : international medical journal of experimental and clinical research 21, 376-382.

Martincich, L., Deantoni, V., Bertotto, I., Redana, S., Kubatzki, F., Sarotto, I., Rossi, V., Liotti, M., 
Ponzone, R., Aglietta, M., et al. (2012). Correlations between diffusion-weighted imaging and breast cancer biomarkers. European radiology 22, 1519-1528.

Mazurowski, M.A., Zhang, J., Grimm, L.J., Yoon, S.C., and Silber, J.I. (2014). Radiogenomic analysis of breast cancer: luminal $B$ molecular subtype is associated with enhancement dynamics at MR imaging. Radiology 273, 365-372.

Montemurro, F., Martincich, L., Sarotto, I., Bertotto, I., Ponzone, R., Cellini, L., Redana, S., Sismondi, P., Aglietta, M., and Regge, D. (2007). Relationship between DCE-MRI morphological and functional features and histopathological characteristics of breast cancer. European radiology 17, 1490-1497.

Nam, S.Y., Ko, E.S., Lim, Y., Han, B.K., Ko, E.Y., Choi, J.S., and Lee, J.E. (2018). Preoperative dynamic breast magnetic resonance imaging kinetic features using computer-aided diagnosis: Association with survival outcome and tumor aggressiveness in patients with invasive breast cancer. Plos one 13, e0195756.

Parekh, V.S., and Jacobs, M.A. (2017). Integrated radiomic framework for breast cancer and tumor biology using advanced machine learning and multiparametric MRI. NPJ breast cancer 3, 43.

Rakha, E.A., Reis-Filho, J.S., Baehner, F., Dabbs, D.J., Decker, T., Eusebi, V., Fox, S.B., Ichihara, S., Jacquemier, J., Lakhani, S.R., et al. (2010). Breast cancer prognostic classification in the molecular era: the role of histological grade. Breast cancer research : BCR 12, 207.

Razek, A.A., Gaballa, G., Denewer, A., and Nada, N. (2010). Invasive ductal carcinoma: correlation of apparent diffusion coefficient value with pathological prognostic factors. NMR in biomedicine 23, 619-623.

Shin, H.C., Roth, H.R., Gao, M., Lu, L., Xu, Z., Nogues, I., Yao, J., Mollura, D., and Summers, R.M. (2016). Deep Convolutional Neural Networks for Computer-Aided Detection: CNN Architectures, Dataset Characteristics and Transfer Learning. IEEE transactions on medical imaging 35, 1285-1298.

Smith, S.M., Nichols, T.E., Vidaurre, D., Winkler, A.M., Behrens, T.E., Glasser, M.F., Ugurbil, K., Barch, D.M., Van Essen, D.C., and Miller, K.L. (2015). A positive-negative mode of population covariation links brain connectivity, demographics and behavior. Nature neuroscience 18, 1565-1567.

Truhn, D., Schrading, S., Haarburger, C., Schneider, H., Merhof, D., and Kuhl, C. (2019). Radiomic versus Convolutional Neural Networks Analysis for Classification of Contrast-enhancing Lesions at Multiparametric Breast MRI. Radiology 290, 290-297.

Wang, C., Subashi, E., Yin, F., and Chang, Z. (2015a). Therapeutic Response Assessment Using a Novel Gray Level Local Power Matrix (GLLPM) in DCE-MRI Texture Analysis: Feasibility Study. Medical physics 42, 3212-3213.

Wang, Z.Y., Yang, W.K., and Ben, X.Y. (2015b). Low-resolution degradation face recognition over long distance based on CCA. Neural Comput Appl 26, 1645-1652.

Westra, C., Dialani, V., Mehta, T.S., and Eisenberg, R.L. (2014). Using T2-weighted sequences to more accurately characterize breast masses seen on MRI. AJR American journal of roentgenology 202, W183-190.

Wirapati, P., Sotiriou, C., Kunkel, S., Farmer, P., Pradervand, S., Haibe-Kains, B., Desmedt, C., Ignatiadis, M., Sengstag, T., Schutz, F., et al. (2008). Meta-analysis of gene expression profiles in breast cancer: toward a unified understanding of breast cancer subtyping and prognosis signatures. Breast cancer research : $B C R$ 10, R65.

Wu, J., Li, B., Sun, X., Cao, G., Rubin, D.L., Napel, S., Ikeda, D.M., Kurian, A.W., and Li, R. (2017). Heterogeneous Enhancement Patterns of Tumor-adjacent Parenchyma at MR Imaging Are Associated with Dysregulated Signaling Pathways and Poor Survival in Breast Cancer. Radiology 285, 401-413. 
Xie, Y.T., Zhang, J.P., Xia, Y., Fulham, M., and Zhang, Y.N. (2018). Fusing texture, shape and deep model-learned information at decision level for automated classification of lung nodules on chest CT. Inform Fusion 42, 102-110.

Yang, Q., Li, L., Zhang, J., Shao, G., Zhang, C., and Zheng, B. (2014). Computer-aided diagnosis of breast DCE-MRI images using bilateral asymmetry of contrast enhancement between two breasts. Journal of digital imaging 27, 152-160.

Zhang, B., Tian, J., Dong, D., Gu, D., Dong, Y., Zhang, L., Lian, Z., Liu, J., Luo, X., Pei, S., et al. (2017). Radiomics Features of Multiparametric MRI as Novel Prognostic Factors in Advanced Nasopharyngeal Carcinoma. Clinical cancer research : an official journal of the American Association for Cancer Research 23, 4259-4269.

Zhao, F., Qiao, L., Shi, F., Yap, P.T., and Shen, D. (2017). Feature fusion via hierarchical supervised local CCA for diagnosis of autism spectrum disorder. Brain imaging and behavior 11, 1050-1060. 